\title{
Identifying training and informational components to develop a psoriasis self- management application
}

\author{
Reza Safdari ${ }^{1}$, Alireza Firoz ${ }^{2}$, Hoorie Masoorian ${ }^{3 *}$ \\ Received: 20 Aug 2016 \\ Published: 1 Oct 2017
}

\begin{abstract}
Background: Psoriasis is a complex disease with lifelong emotional and social consequences for affected patients. It also reduces the patients' quality of life and requires a long-term management. Therefore, in addition to appropriate treatment of the disease, selfmanagement strategies to improve patient health and quality of life are essential. On the other hand, smartphone-based applications alter the way people interact with health care and public health systems. This study aimed at identifying training and informational components to develop a psoriasis self- management application.

Methods: This descriptive-analytic study was conducted on 100 patients with psoriasis and 26 dermatologists who were selected randomly, using Morgan table. The data were collected using a researcher- made questionnaire, which included demographic and clinical information, lifestyle training and management, and application capabilities in psoriasis self-management. A group of experts and a test-retest method were used to confirm the validity and reliability of the questionnaire, respectively.

Results: The mean scores for demographic and clinical information, lifestyle training and management, and application capabilities in self-management were $80.55 \%, 85.7 \%$, and $88.8 \%$ from the patients' perspective, and $83.7 \%, 71 \%$, and $75 \%$ from the specialists' viewpoint, respectively.

Conclusion: Determining self-management components by patients as persons who are suffering from the disease and physicians as specialists in the field will be helpful in efficient psoriasis self-management. It is more likely that self-reliant patients, who are aware of the benefits and risks of their disease management application, will follow their treatment plan and pursue the management of their disease more seriously.
\end{abstract}

Keywords: Psoriasis, Self-management, Application

Copyright $\odot$ Iran University of Medical Sciences

Cite this article as: Safdari R, Firoz A, Masoorian H. Identifying training and informational components to develop a psoriasis self- management application. Med J Islam Repub Iran. 2017 (1 Oct);31:67. https://doi.org/10.14196/mjiri.31.67

\section{Introduction}

The historical study of the diseases in the last century shows a trend away from infectious and noncommunicable towards chronic and non-infectious diseases (1). Chronic diseases are the leading cause of morbidity and mortality worldwide, accounting for $63 \%$ of all deaths and $43 \%$ of the global burden of disease (2). An increased number of individuals with chronic diseases is a signifi-

\section{Corresponding author: Hoorie Masoorian, hmasoorian@gmail.com}

1. Health Information Department, Health Information Management Research Center, School of Allied Medical Sciences, Tehran University of Medical Sciences, Tehran, Iran.

2. Center of Research and Training in Skin and Leprosy, Tehran University of Medical Sciences, Tehran, Iran.

3. School of Allied Medical Sciences, Tehran University of Medical Sciences, Tehran, Iran. cant clinical and economic challenge for health care systems worldwide (3).

Psoriasis is a chronic inflammatory skin disease, affecting approximately $0 \%$ to $11.8 \%$ of the world's population (4). It affects both males and females almost equally and can occur at any age (5). The main manifestations include scaling erythematous plaques resulting in inflammation

$\uparrow$ What is “already known” in this topic:

Psoriasis is a chronic inflammatory skin disease, and it was affected to several organs of body and caused many problems for patient. It also reduces the patients' quality of life and requires a long-term management.

$\rightarrow$ What this article adds:

This study, found and gathered the most important elements of self-management of Psoriasis by needing assessment from patients (as an user of application) and dermatologists (as an expert) to design and establish psoriasis self-management application. 
and pain, affecting quality of life (6). In addition to skin, psoriasis affects several other systems in the body. This disease increases the risk of comorbid conditions due to the resulting immune disorders and subsequent inflammation (7) and reduces health-related quality of life (HRQOL) (6). Studies show that reduction of HRQOL by psoriasis is similar to other chronic diseases such as diabetes, ischemic heart disease, and cancers (8). Psoriasis is a complex disease with lifelong emotional and social consequences, which decreases the quality of life and requires long-term management (9). In addition to an appropriate treatment, self-management strategies to enhance patients' health and improve the quality of life are essential (10).

Self-management programs are widely used for empowerment, increasing self-confidence, training skills and techniques, and improving interaction with the health care system to better manage chronic diseases (11). It is also defined as patients' ability to manage the symptoms, treat physical and psychological consequences, and modify lifestyle to cope with chronic diseases to reduce their impact on daily life, increase the overall quality of life, enhance medication adherence, ensure adequate sleep, maintain a healthy diet, reduce stress, establish mental balance, reduce dependence on other people, and cope with the shame-inducing experiences $(10,12)$.

In recent years, the relationship between diseases, physical activity, and lifestyle parameters such as smoking, stress, and body mass index has been identified as areas which can positively or negatively be affected by psoriasis. Thus, training patients and performing selfmanagement interventions seem particularly important for patients with psoriasis (13). Successful psoriasis selfmanagement requires empowerment of patients to face the disease and address its medical and psychological aspects. Patients should be empowered through training and seeking support for both medical and psychological aspects of their disease. Successful psoriasis self-management can be achieved through a multidimensional approach (14).

More recently, efforts have been made to develop new models for chronic disease management using information technology. In particular, mobile health interventions have a positive impact on chronic disease management. Smart phones have been introduced as one of the most promising tools because of their widespread and convenient use (15).

Mobile-based interventions have advantages compared with face to face approaches (16). For example, chronic disease management applications, which combine mobile and web-based technologies, can potentially change the focus from clinic to daily life, in which behavior change is realized (17).

Because the prevalence of psoriasis is estimated $1.3 \%$ to $2.5 \%$ in Iran (18), no study has been conducted to identify training and informational needs of patients with psoriasis with respect to self-management of the disease, and only treatment needs of these patients have been examined in most cases. Thus, the present study aimed at determining training and informational needs for psoriasis selfmanagement to develop an application according to patient and physician viewpoints.

\section{Methods}

\section{Literature Review}

A review of literature and relevant academic references was performed to extract factors influencing psoriasis selfmanagement and provide the required scientific evidence related to the above- mentioned components. Accordingly, electronic databases such as Scopus, Web of Science, Science Direct, and PubMed, as well as library references were searched from 2005 to 2016. Figure 1 depicts the selection process of citation sources in the literature review stage.

\section{Reference Search}

Keywords searched for scientific references included "psoriasis self-management" and "psoriasis self-care" in combination with "dataset", "needs assessment", and "education" through the operator "AND". The search was limited to titles and abstracts, English and Persian resources, and the time interval of 2005 to 2016.

\section{The First Edition of the Components List from the Spe-} cialist Viewpoint

According to the viewpoints of 2 dermatologists, a modified list of self-management for training and intervention was prepared.

\section{Sample Size}

We assigned a total of 100 psoriasis patients aged 10 to 65 years who were registered in Psoriasis Association. All participants provided written informed consent. Patients were eligible if they were literate and had smart phones.

Then, 15 dermatologists from hospitals affiliated to Tehran University of Medical Sciences (TUMS) and 11 from Center for Research and Training in Skin Diseases and Leprosy of (TUMS) were also randomly selected. The only inclusion criterion for dermatologists was their specialty.

The study objectives were explained to the participants and they were assured of information confidentiality and their right to withdraw at any time.

\section{Designing Data Collection Tool}

In this study, 2 questionnaires for physicians and patients were separately developed by the researcher to determine the most important information and the required components of the psoriasis self-management Android application.

In addition to the demographic information of the participants including gender, age, education, marital status, place of birth, occupation, and age at onset of the disease, the patient questionnaire had 3 main sections: (1) demographic and clinical information; (2) training and lifestyle management; and (3) application capabilities adjusted by patients according to their needs for managing their condition. In total, there were 50 questions in the questionnaire, with 19 questions in the first section, 22 in the second, and 9 in the third section.

In addition to identity information including gender, age, qualification, and experience, the physicians' questionnaire had sections similar to those of the patients' plus 
Identifying articles

Appropriateness of articles

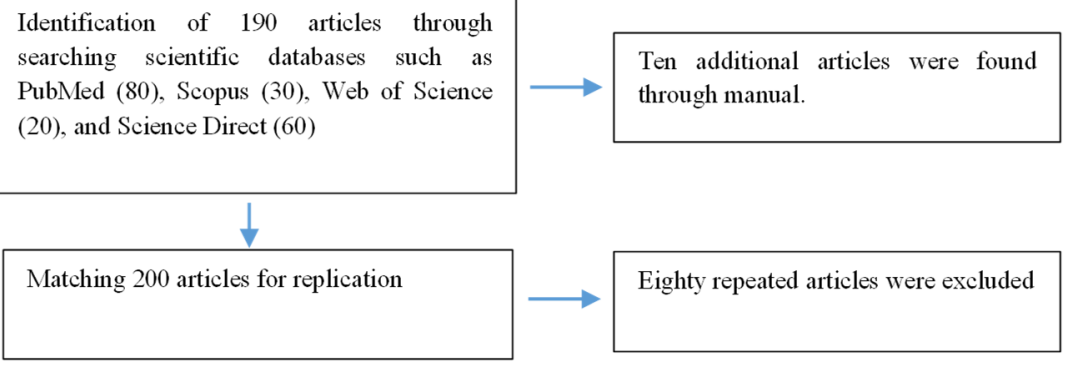
searching scientific databases such as

PubMed $(80)$, Scopus $(30)$, Web of Science

(20), and Science Direct $(60)$
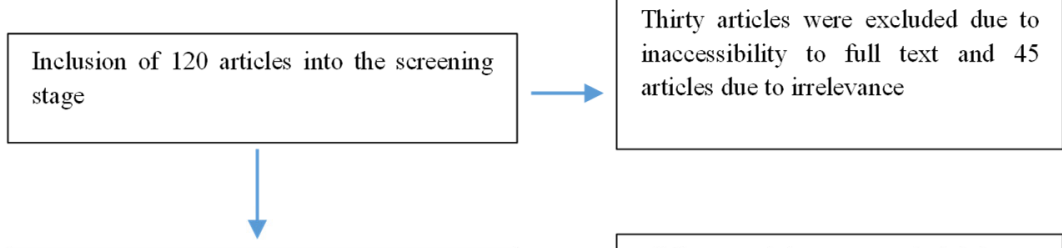

Review of 45 articles for appropriateness

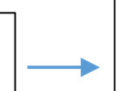

Eighteen articles were excluded due to not focusing on the subject

Fig. 1. The Selection Process of Citation Sources in the Literature Review Stage

educational items about the treatment techniques. The first, second, and third sections included 16, 38, and 9 questions, respectively (63 questions).

\section{Validation of the Selected Key Components Using the Delphi Method}

To validate the extracted key components, we provided the components to the participants (patients and physicians) in the form of Delphi process. In the first stage of the Delphi process, the participants were asked to determine the relative importance of each proposed component with respect to the psoriasis self-management using a questionnaire (a 5-point Likert scale) and to suggest their desired components not mentioned in the list.

\section{Validity and Reliability}

The validity of the questionnaire was confirmed by 5 dermatologists and information managers. Test-retest was used to determine the reliability of the questionnaire, so that it was completed by 30 patients and 5 physicians on 2 occasions, with a 7- day interval; finally, the reliability of the questionnaire was approved by Cronbach's alpha of $90 \%$.

\section{Data Collection}

Due to the use of Likert scale, the data were collected on 2 occasions. First, the questionnaires were distributed in person among physicians and patients. After analyzing the data, the components not gaining an acceptable score according to Table 1, were studied in the second stage in which the questionnaires were sent to the physicians and patients through e-mail, and the responses were received after a week.

\section{Data Analysis}

Data were analyzed and the confidence intervals (CI) were calculated using the SPSS-16 software program.

\section{Results}

\section{Findings from the Patients' Viewpoint}

The frequency and demographic data of the patients are presented in Table 2.

According to Table 2, the percentage of male and female participants is close to each other, so it shows that the prevalence of psoriasis is the same between males and females. The level of education and the job status of patients were meaningless. This may be due to the reluctance of patients to enter in the society and continue their education. It can be claimed that the disease has an effect on marital status and half of the participants were single.

The results (mean score and accepted components after 2 stages of needs assessment) of the second section of the questionnaire are provided in Fig. 2 (the percent of each section as a whole) and in Tables 3 and 4 (the percent of

\section{Table 1. Criteria for Selecting the Components}

\begin{tabular}{cc} 
Mean/median score $>3.75$ & Acceptance of the index in the first stage of Delphi \\
Mean/median score $2.5-3.75$ & Review in the second stage of Delphi \\
Mean/median score $<2.5$ & Rejection of the index in the first stage of Delphi \\
\hline
\end{tabular}




\begin{tabular}{llcc}
\multicolumn{3}{l}{ Table 2. Frequency and Demographic Data of the Patients } \\
\hline Variable & & Number & Frequency \\
\hline Sex & Female & 47 & $47 \%$ \\
& Male & 53 & $53 \%$ \\
& $10-1410 / 10$ & 3 & $2 \%$ \\
Age & $15-2410 / 10$ & 23 & $22 \%$ \\
(year) & $25-3410 / 10$ & 42 & $42 \%$ \\
& $35-4410 / 10$ & 17 & $17 \%$ \\
Type of & $45-65$ & 17 & $17 \%$ \\
qualification & High school diploma & 3 & $3 \%$ \\
& Diploma & 39 & $29 \%$ \\
& Associate Degree & 9 & $9 \%$ \\
& Bachelor & 43 & $42 \%$ \\
Marital & Masters & 18 & $18 \%$ \\
status & Single & 45 & $45 \%$ \\
Job type & Married & 55 & $55 \%$ \\
& Self-employed & 35 & $35 \%$ \\
& Government job & 28 & $28 \%$ \\
& Unemployed & 37 & $37 \%$ \\
\hline
\end{tabular}

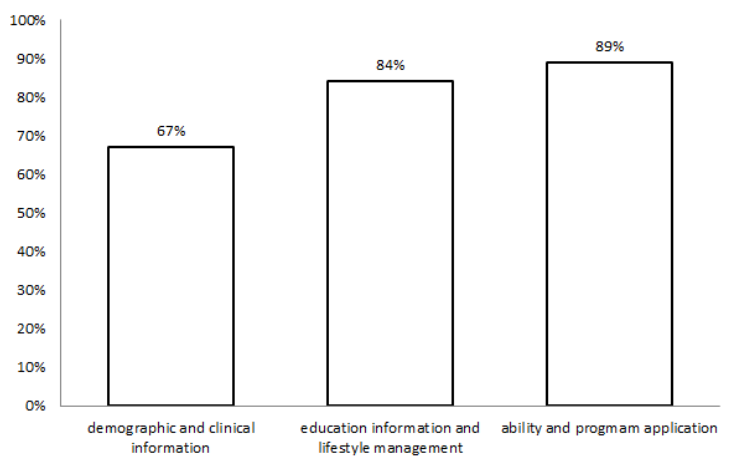

Fig. 2. Findings of the Second Section of the Questionnaire (the percent of each section)

details of each section in detail).
Patients confirmed the need of the section "ability and applications" more than other parts of the questionnaire, indicating the tendency of patients to perform self-control and self-management of their disease.

According to Table 4, the percentage of the patients' different needs with respect to the content of categories revealed the importance of medication for the psoriasis treatment. The patients assigned a high score to type of drug in section "demographic and clinical information", drug treatment in section "educational information and lifestyle management" and the reminder of drug in section "ability and application".

\section{Findings from the Physicians' Viewpoint}

The frequency and demographic data of the physicians are presented in Table 5.

According to Table 5, 70\% of physicians participating in the study were 30 to 49 years old, indicating the greater willingness of young physicians to cooperate in research studies. On the other hand, most of the physicians in the study were experts and had more than 20 years of work experience, which helps design a more efficient program.

The second part of the questionnaire is provided in Fig. 3 (the percent of each section) and in Tables 6 and 7 (the percent of details of each section).

Physicians, who participated in this study, confirmed the importance of the section "education information and lifestyle management" more than other parts of the questionnaire.

According to the table, the percent of the importance of content of categories from the perspective of dermatologists indicates the importance of treatment of psoriasis using drug and depression management. The physicians

Table 3. The First Stage of Needs Assessment of Patients (components with enough score)

\begin{tabular}{|c|c|c|c|}
\hline Axis & Variable & Average Rate & $\mathrm{CI}$ \\
\hline \multirow{12}{*}{$\begin{array}{l}\text { Demographic and clinical } \\
\text { information. }\end{array}$} & Age (Year of birth) & 3.82 & $3.32-4.32$ \\
\hline & Sex & 3.9 & $3.4-4.4$ \\
\hline & Weight & 3.75 & $3.25-4.25$ \\
\hline & History of psoriasis (family / relatives) & 4.04 & $3.54-4.54$ \\
\hline & The average monthly income & 3.92 & $3.42-4.42$ \\
\hline & Insurance cover & 3.99 & $3.49-4.49$ \\
\hline & First signs of psoriasis in age (day / month / year) & 4.02 & $3.52-4.52$ \\
\hline & Detection time of psoriasis disease & 4.02 & $3.52-4.52$ \\
\hline & After the first symptoms of psoriasis (day / month / year) & & \\
\hline & The first site of disease (palms, soles of the feet, extensor parts, genitals, scalp, etc.) & 4.20 & $3.7-4.7$ \\
\hline & The type of treatment used had & 4.25 & $3.78-4.78$ \\
\hline & Infected with diseases other than psoriasis & 4.29 & $3.79-4.79$ \\
\hline \multirow{19}{*}{$\begin{array}{l}\text { Educational information } \\
\text { and lifestyle management }\end{array}$} & Type drugs & 4.31 & $3.81-4.81$ \\
\hline & Forms of psoriasis & 4.36 & $3.86-4.86$ \\
\hline & Symptoms of Psoriasis & 4.37 & $3.87-4.87$ \\
\hline & The causative factors of psoriasis & 4.95 & $4.45-5.45$ \\
\hline & A variety of detection methods Psoriasis & 4.46 & $3.96-4.96$ \\
\hline & Treatment of psoriasis & 4.43 & $3.93-4.93$ \\
\hline & A variety of Comorbidities of psoriasis & 4.45 & $3.95-4.95$ \\
\hline & Drug treatment & 4.91 & $4.41-5.41$ \\
\hline & Traditional medicine & 4.33 & $3.83-4.83$ \\
\hline & Treatment of mental and physical & 4.25 & $3.75-4.75$ \\
\hline & Alternative therapies (acupuncture) & 4.29 & $3.79-4.79$ \\
\hline & Other complementary therapies (such as home remedies) & 4.12 & $3.62-4.62$ \\
\hline & Phototherapy & 4.02 & $3.52-4.52$ \\
\hline & (nutrition): No food restrictions (Normal) & 3.65 & $3.15-4.15$ \\
\hline & (Nutrition) :Seafood & 4.27 & $3.77-4.77$ \\
\hline & (physical activity) :Aerobic exercise & 4.12 & $3.62-4.62$ \\
\hline & (physical activity): Yoga & 4.04 & $3.54-4.54$ \\
\hline & (physical activity) :Meditation & 4.56 & $4.06-5.06$ \\
\hline & Stress Management (positivism) & 4.77 & $4.27-5.27$ \\
\hline
\end{tabular}




\begin{tabular}{|c|c|c|c|}
\hline \multirow{9}{*}{$\begin{array}{l}\text { Ability and } \\
\text { program } \\
\text { applications }\end{array}$} & Notices: The main medical centers and dealing with psoriasis & 4.56 & $4.06-5.06$ \\
\hline & Notices: List dermatologist & 4.61 & 4.11-5.12 \\
\hline & (Reminder) of the need for time-consuming drugs & 4.44 & $3.94-4.94$ \\
\hline & (Reminder) of the need to visit a doctor's office & 4.39 & $3.89-4.89$ \\
\hline & (Reminder) necessary to perform specific exercises for Disease Control and overcome stress & 4.38 & $3.88-4.88$ \\
\hline & (Reminder) necessary to carry out targeted feeding & 4.38 & $3.88-4.88$ \\
\hline & Set Goal: Axis feed & 4.49 & $3.99-4.99$ \\
\hline & Set Goal: Axis of physical activity & 4.47 & $3.97-4.97$ \\
\hline & Encouraging and soothing words for (notifications on mobile) daily / weekly & 4.25 & $3.75-4.75$ \\
\hline
\end{tabular}

Table 4. The Second Stage of Needs Assessment of Patients (Components gained the required score in the second stage.)

\begin{tabular}{|c|c|c|c|}
\hline Axis & Variable & Average Rate & $\mathrm{CI}$ \\
\hline \multirow{2}{*}{ Demographic and clinical information } & Height & 3.85 & 3.35 \\
\hline & (Physical activity): Massage & 3.92 & $3.42-4.42$ \\
\hline \multirow{2}{*}{$\begin{array}{l}\text { Educational information } \\
\text { and lifestyle management }\end{array}$} & (Nutrition): Vegetarian & 3.98 & $3.48-4.48$ \\
\hline & (Nutrition): Vegetarianism & 3.74 & $3.24-4.24$ \\
\hline
\end{tabular}

\begin{tabular}{|c|c|c|c|}
\hline Variable & & Number & Frequency \\
\hline \multirow[t]{2}{*}{ Sex } & Female & 12 & $46 \%$ \\
\hline & Male & 14 & $54 \%$ \\
\hline Age & $30-4910 / 10$ & 19 & $71 \%$ \\
\hline \multirow[t]{2}{*}{ (year) } & $50-7010 / 10$ & 6 & $24 \%$ \\
\hline & +70 & 1 & $5 \%$ \\
\hline \multirow[t]{3}{*}{ Type of qualification } & Specialty & 4 & $12 \%$ \\
\hline & Expertise & 20 & $76 \%$ \\
\hline & Resident & 2 & $8 \%$ \\
\hline \multirow[t]{4}{*}{ The work experience (years) } & $1-910 / 10$ & 5 & $20 \%$ \\
\hline & $10-1910 / 10$ & 4 & $15 \%$ \\
\hline & $20-3010 / 10$ & 10 & $38 \%$ \\
\hline & +30 & 7 & $27 \%$ \\
\hline
\end{tabular}

assigned a high score to history of disease in the following section.

s: "demographic and clinical information"; disease treatment and depression management in section "educational information and lifestyle management"; and reminder of drugs in section "ability and application".

\section{Discussion}

Chronic skin diseases such as psoriasis have a profound negative impact on quality of life, and thus require longterm management. Therefore, training and motivating play an important role in long-term management of chronic diseases. Successful training increases patients' satisfac-

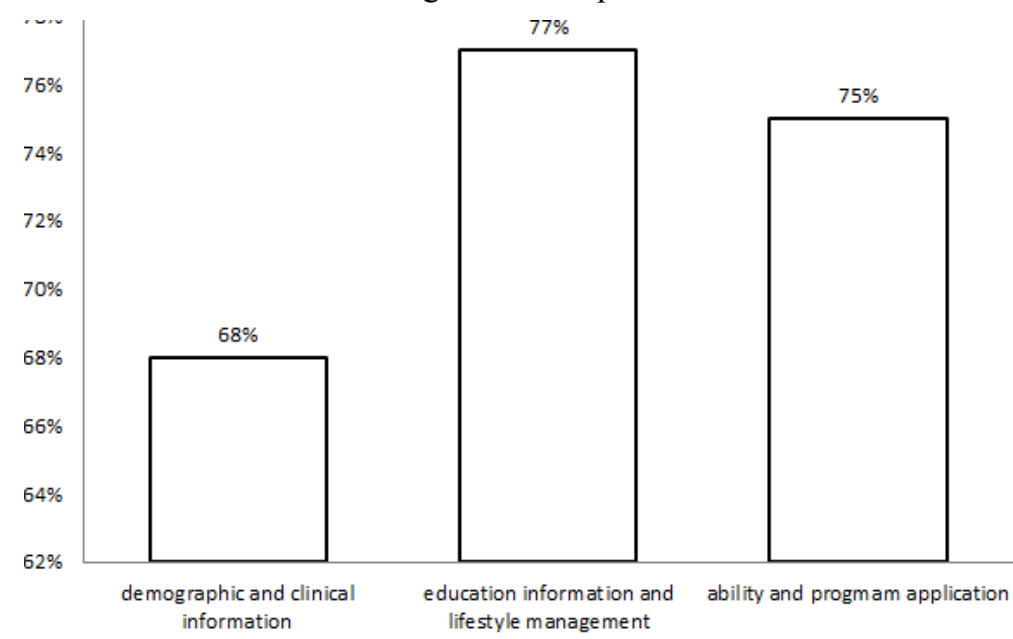

Fig. 3. Findings of the Second Section of the Questionnaire (the percent of each section) tion and improves health and treatment adherence (14). High scores given by patients and physicians to selfmanagement components, in which training is the pivotal aspect, highlight the importance of this issue.

In the clinical and demographic information section, the most important components regarding the perspective of the patients included the history of psoriasis, suffering from other diseases, and the medications with the high average score of 4 . Regarding the perspective of the experts in this study, the history of psoriasis, suffering from other diseases, and age at onset of psoriasis had the high average score of 4 . These findings are consistent with the study of JL López-Estebaranz et al. (2015), in which the 


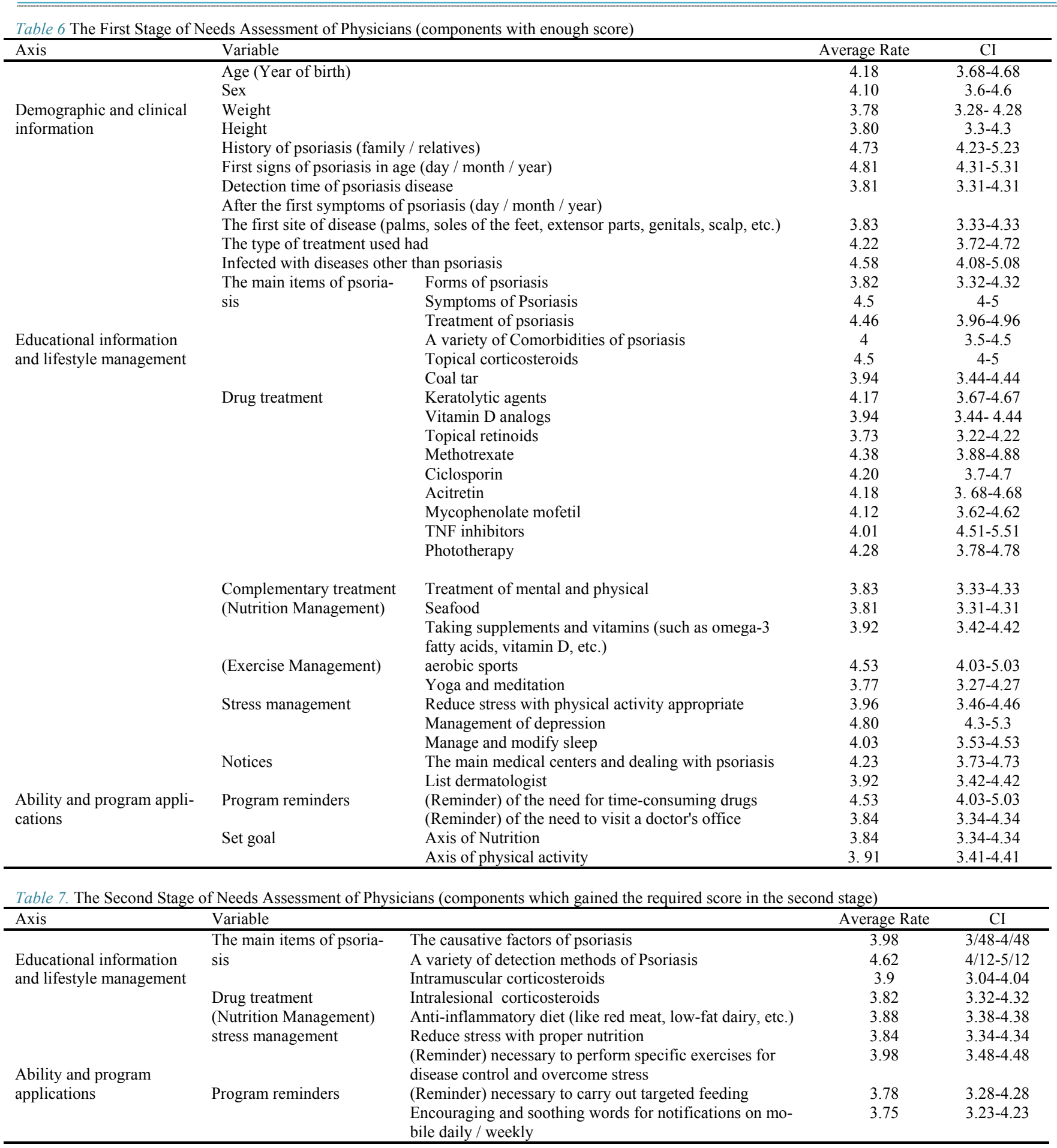

importance and impact of family history and age on patients with psoriasis and their quality of life were moderate to severe (19).

Considering the scores of needs assessment, the results revealed that patients with psoriasis obtained a high average score of 4 in all areas of training and lifestyle management. Physicians paid particular attention to all informational parts of the application, with the average score of 3.75. Patients' awareness of different aspects of their condition affected their opinion. These findings are consistent with the study of Padmavathi Nagarajan (2016) who stated that all efforts should be done to fully inform patients about their condition and make them master in factors that can help self-care (20).

This study revealed that patients and physicians gave high scores to the fields of training including the main items of the disease and its various treatments, either pharmacological or non-pharmacological, with a mean score of 3.75 , indicating the inadequate training information level of people. This finding is in line with the study of Skiveren et al. who found that patients' awareness about the risk of concomitant diseases such as metabolic syndrome is low and they need to be trained about the disease (21). 
In the lifestyle management section, 3 parts of nutritional, exercise, and stress management were important from the viewpoints of patients and physicians with an average score of 3.8. Therefore, stress management was of high priority and importance from the viewpoints of patients and physicians with a high average score of 4 , necessitating further training.

Depression management is an approach to manage stress, and both physicians and patients stated the importance of its training which is consistent with the study of Korman et al. (2016) who expressed the importance of nonpharmacological treatments of depression in selfmanagement. These treatments include cognitive behavioral therapy and other techniques used for reducing stress, achieving promising results, reducing anxiety, depression, and stress, and reducing psoriatic lesions (22). Ayman Elgendi et al. (2015) pointed out the significant reduction of depression symptoms following psoriasis improvement and suggested screening and recognition of depression symptoms as a main part of psoriasis management due to high prevalence of this disorder in these patients (23).

Relaxation techniques for stress relief are among the psoriasis self-management methods. Meditation belongs to this group of therapy, and physicians emphasize the importance of physical activity and subsequently stress management to manage psoriasis, with a high average score of 4. Nagarajan Padmavathi et al. (2014) also pointed out the improvement of psoriasis through stress management techniques such as relaxation treatments (meditation and yoga) (24).

Proper nutrition and consumption of vitamins and supplements are helpful in treating psoriasis. Specialists have considered the importance of vitamins with a score of 4; this is in line with the study of T Soleymani et al. (2015), according to whom, the role of vitamins, especially vitamin $\mathrm{D}$, is very complex, extensive, and effective in psoriasis management (25).

In addition, the components related to capabilities of the application from the patient and physician viewpoints received a high mean score of 4 . Since the above- mentioned components emphasize more on increasing the interactivity of the application the user deals with, this high score shows that both patients and physicians believe that increased user-application interaction will improve the disease self-management. This finding is in line with the study of Da Tao et al. (2014) who stated that the use of electronic reminders for chronic patients care and increased treatment adherence is important and effective (26).

\section{Conclusion}

Developing a smart phone application for psoriasis selfmanagement requires identifying disease self-management components using the patients and physicians' viewpoints. Given that the end users involvement in application design will increase its effectiveness and efficiency, taking into account the viewpoints of the patients as the system end users and the physicians as specialists in the field will make this application effective. In addition, identifying self-management components from the patient and specialist viewpoints and developing a psoriasis selfmanagement application will undoubtedly help better control and manage the disease.

\section{Acknowledgement}

The Authors wish to thank the staff of Center for Research and Training in Skin Diseases and Leprosy and School of Allied Medicine, Tehran University of Medical Sciences for their generous help.

\section{Conflict of Interests}

The authors declare that they have no competing interests.

\section{References}

1. Bokemeyer B, Hardt J, Hüppe D, Prenzler A, Conrad S, Düffelmeyer $M$, et al. Clinical status, psychosocial impairments, medical treatment and health care costs for patients with inflammatory bowel disease (IBD) in Germany: an online IBD registry. J. Crohns Colitis. 2013;7(5):355-68.

2. Nes AA, Eide H, Kristjánsdóttir ÓB, van Dulmen S. Webbased, self-management enhancing interventions with ediaries and personalized feedback for persons with chronic illness: A tale of three studies. Patient Educ Couns. 2013;93(3):451-8

3. Gibbons KJ. Apnea testing: An effective and safer means. Crit Care Med 2006;34(8):2257.

4. Gupta R, Debbaneh MG, Liao W. Genetic epidemiology of psoriasis .Curr Dermatol Rep. 2014;3(1):61-78.

5. Korkoliakou P, Christodoulou C, Kouris A, Porichi E, Efstathiou V, Kaloudi E, et al. Alexithymia, anxiety and depression in patients with psoriasis: a case-control study. Ann Gen Psychiatry. 2014;38:13-43.

6. Pickett K, Loveman E, Kalita N, Frampton G, Jones J. Educational interventions to improve quality of life in people with chronic inflammatory skin diseases: systematic reviews of clinical effectiveness and cost-effectiveness. HTA. 2015;19(86):3-6.

7. Feldman SR, Zhao Y, Shi L, Tran MH. Economic and Comorbidity Burden Among Patients with Moderate-toSevere Psoriasis. J Manag Care Spec Pharm. 2015;21(10):874.

8. Farahangiz S, Hadi N, Naseri M, Agah E, Montazeri A. Assessment of Health-Related Quality of Life in Patients With Psoriasis in Comparison With Normal Subjects in Shiraz, Iran. SEMJ. 2014;15(2).

9. Hamilton M, Ntais D, Griffiths C, Davies L. Psoriasis treatment and management-a systematic review of full economic evaluations. Br. J. Dermatol.. 2015;172(3):574-83.

10. Bautista RED, Shoraka AR, Shapovalov D. Factors associated with superior self-management skills among individuals with epilepsy. Epilepsy Behav. 2014;221:41-6.

11. Jaglal SB, Guilcher SJ, Hawker G, Lou W, Salbach NM, Manno M, et al. Impact of a chronic disease self-management program on health care utilization in rural communities: a retrospective cohort study using linked administrative data .BMC Health Serv. Res.. 2014;14(1):198.

12. Escoffery C, Bamps Y, LaFrance WC, Stoll S, Shegog R, Buelow J, et al. Factor analyses of an Adult Epilepsy SelfManagement Measurement Instrument (AESMMI). Epilepsy Behav. 2015;184:50-9.

13. Larsen MH, Hagen KB, Krogstad AL, Aas E, Wahl AK. Limited evidence of the effects of patient education and selfmanagement interventions in psoriasis patients: a systematic 
review Patient Educ Couns. 2014;94(2):158-69.

14. Pathak SN. Self-management in patients with psoriasis. Dovepress. 2014.

15. Kim YJ, Rhee SY, Byun JK, Park SY, Hong SM, Chin SO, et al. A Smartphone Application Significantly Improved Diabetes Self-Care Activities with High User Satisfaction J Diabetes Metab. 2015;39.

16. Smith C, Ngo TD, Gold J, Edwards P, Vannak U, Sokhey L, et al. Effect of a mobile phone-based intervention on postabortion contraception: a randomized controlled trial in Cambodia. Bulletin of WHO. 2015;93(12):842-50.

17. Harris LT, Tufano J, Le T, Rees C, Lewis GA, Evert AB ,et al. Designing mobile support for glycemic control in patients with diabetes. J Biomed Inform. 2010;43(5):37-40.

18. Fanni Rencz M, Ahmad Moradi M, Gulácsi L. Health Status and Quality of Life in Patients with Psoriasis: An Iranian Cross-Sectional Survey. Archives of Iranian medicine. 2015;18(3):153.

19. Balato N, Megna M, Di Costanzo L, Balato A, Ayala F. Educational and motivational support service: a pilot study for mobile-phone-based interventions in patients with psoriasis. Br J Dermatol. 2013;168(1):201-5.

20. Nagarajan P, Karunagari K, Thappa D. A Questionnairebased Survey of Patients' Knowledge Regarding Psoriasis. Arch Psychiatr Nurs. 2016;2(1):18-23.

21. Skiveren J, Philipsen P, Therming G. Patients with psoriasis have insufficient knowledge of their risk of atherothrombotic disease and metabolic syndrome. Clin Exp Dermatol. 2015;40(6):600-4.

22. Toledo-Pastrana T, García-Hernández MJ, CarrizosaEsquivel AM, Camacho-Martínez FM. Evaluation of 25 years of phototherapy for treating psoriasis at a teaching hospital in southern Spain. An Bras Dermatol. 2015;90(4):473-8.

23. Gazi S, Ali S, Talmale S, Pange S, Kadam B, Surwase U. Quality of life in pateints with psoriasis in osmanabad region of india: a single centre study. Int J Pharm Sci Res. 2014;5(10):4522.

24. Thorlund K, Druyts E, Aviña-Zubieta JA, Mills EJ. Antitumor necrosis factor (TNF) drugs for the treatment of psoriatic arthritis: an indirect comparison meta-analysis. Biologics. 2012;417:6-12.

25. Ersser S, Cowdell F, Latter S, Healy E. Self-management experiences in adults with mild-moderate psoriasis: an exploratory study and implications for improved support. Br. J. Dermatol. 2010;163(5):1044-9.

26. O’Leary K, Vizer L, Eschler J, Ralston J, Pratt W, editors. Understanding patients' health and technology attitudes for tailoring self-management interventions. AMIA Annual Symposium Proceedings. J Am Med Inform Assoc. 2015. 